\title{
Tumor-infiltrating neutrophils in pancreatic neoplasia
}

\author{
Michelle D Reid ${ }^{1}$, Olca Basturk ${ }^{2}$, Duangpen Thirabanjasak ${ }^{3}$, Ralpha H Hruban ${ }^{4}$, \\ David S Klimstra ${ }^{2}$, Pelin Bagci ${ }^{1}$, Deniz Altinel ${ }^{1}$ and Volkan Adsay ${ }^{1}$ \\ ${ }^{1}$ Department of Pathology, Emory University, Atlanta, GA, USA; ${ }^{2}$ Department of Pathology, Memorial \\ Sloan-Kettering Cancer Center, New York, NY, USA; ${ }^{3}$ Department of Pathology, Chulalongkorn University, \\ Thailand and ${ }^{4}$ Department of Pathology, The Sol Goldman Pancreatic Cancer Research Center, \\ Johns Hopkins University, Baltimore, MD, USA
}

The interaction between tumor cells and inflammatory cells has an important role in cancer initiation and progression; however, this interaction has not been systematically investigated in pancreatic neoplasia. In this study, the presence of tumor-infiltrating neutrophils within and/or adjacent to neoplastic cells was investigated in pancreatic neoplasms. Areas with $>10$ tumor-infiltrating neutrophils/100 epithelial cells were arbitrarily classified as positive. Those with 11-15 tumor-infiltrating neutrophils were considered 'borderline' while those with $>15$ tumor-infiltrating neutrophils were considered 'significant'. Among 363 invasive ductal carcinomas, 15 showed significant tumor-infiltrating neutrophils (8 were micropapillary carcinomas and 7 were undifferentiated carcinomas). Of 19 mucinous cystic neoplasms with a carcinomatous high-grade papillary component, 11 showed significant tumor-infiltrating neutrophils (mean, 25; range, 14-63 tumor-infiltrating neutrophils). Among intraductal papillary mucinous neoplasms, significant tumor-infiltrating neutrophils were identified in 4/16 pancreatobiliary type, but were uncommon in other types (1/11 oncocytic and 1/23 intestinal types had borderline tumor-infiltrating neutrophils, and 0/10 gastric type had tumor-infiltrating neutrophils). Non-carcinomatous (low-grade and non-papillary) components of these neoplasms did not have tumorinfiltrating neutrophils. Tumor-infiltrating neutrophils were not striking in neuroendocrine tumors (40), serous cystadenomas (18), acinar cell carcinomas (9) or solid-pseudopapillary neoplasms (8). In conclusion, significant tumor-infiltrating neutrophils are uncommon in pancreatic ductal adenocarcinoma, and when they occur it is typically in the micropapillary and undifferentiated types with a known poor prognosis. Among pre-invasive neoplasia, tumor-infiltrating neutrophils show a predilection for papillary in-situ carcinomas of mucinous cystic neoplasms, or less commonly, pancreatobiliary-type intraductal papillary mucinous neoplasms (both of which express cell surface-associated mucin 1 (MUC1)). MUC1 expression by these tumors may have biologic implications, considering its recently established relationship with inflammatory cells in carcinogenesis, and the differential expression of mucins in pancreatic neoplasia. Larger studies are needed to investigate the association between tumor-infiltrating neutrophils and pancreatic neoplasms and their role in their clinical behavior.

Modern Pathology (2011) 24, 1612-1619; doi:10.1038/modpathol.2011.113; published online 5 August 2011

Keywords: neoplasms; pancreatic; tumor-infiltrating neutrophils

Correspondence: Professor V Adsay, MD, Department of Pathology, Emory University Hospital and Winship Cancer Institute, 1364 Clifton Rd. NE, Room H-180B, Atlanta, GA 30322, USA.

E-mail: volkan.adsay@emory.edu

This study was presented in part at the annual meeting of the United States and Canadian Academy of Pathology in Denver in 2008.

Received 10 October 2010; revised 8 June 2011; accepted 8 June 2011; published online 5 August 2011
The importance of the interaction between neoplastic cells and inflammatory cells in cancer initiation and progression is becoming increasingly recognized. This interaction is however not fully understood, and the effect of neutrophils on neoplastic cells seems to represent opposite sides of a doubleedged sword. In some experimental tumors, neutrophils are cytolytic and eliminate neoplastic cells through a variety of factors, ${ }^{1-3}$ including antibodydependent cell-mediated cytotoxicity, whereas in 
others, neutrophils contribute to invasive potential by promoting angiogenesis and basement membrane degradation during extravasation. ${ }^{4-6}$ Therefore, the presence of tumor-infiltrating neutrophils might be indicative of a better or worse hostantitumoral response, which encourages further investigation.

As a result, increasing numbers of researchers have been studying the presence of neutrophils and other inflammatory cells such as mast cells and eosinophils in human cancers ${ }^{1,4-13}$ including gastric, ${ }^{8,10}$ colorectal, ${ }^{13}$ mammary ${ }^{4,5,7,14}$ and bronchioloalveolar carcinomas, ${ }^{6,11}$ gliomas, ${ }^{9}$ lymphomas ${ }^{15}$ and melanoma, ${ }^{15}$ as well as the possible link between inflammatory cells and other parameters with wellknown markers of prognosis. Although, tumorinfiltrating neutrophils were not found to be significantly associated with prognosis in some of these studies, ${ }^{13}$ they correlated with higher tumor grade $^{9}$ or shorter survival in others. In an earlier study, we also noted an association between invasive micropapillary carcinoma of the pancreas and ampullary/periampullar region and tumorinfiltrating neutrophils. ${ }^{16}$ However, a systematic review of tumor neutrophilia and its association with pancreatic neoplasms has not been previously published.

In this study, the presence of, and possible association between, tumor neutrophilia and various histological types of pancreatic neoplasms was systematically investigated.

\section{Materials and methods}

This study was conducted with the approval of and in accordance with the Institutional Review Boards of Wayne State University, Johns Hopkins University, Memorial Hospital for Cancer and Allied Diseases, and Emory University.

\section{Case Selection}

A large series of randomly selected pancreatic neoplasms including 340 usual ductal carcinomas, 8 micropapillary carcinomas, 7 undifferentiated carcinomas, 14 colloid carcinomas, 9 acinar cell carcinomas, 19 mucinous cystic neoplasms with papillary carcinoma in-situ component (all showing pancreatobiliary phenotype), 60 intraductal papillary mucinous neoplasms with predominant papillary component (23 intestinal, 16 pancreatobiliary, 11 oncocytic and 10 gastric type), 18 serous cystadenomas, 40 neuroendocrine neoplasms and 8 solid-pseudopapillary neoplasms were retrieved from the archives of the authors' institutions. These pancreatic tumors represent the same case population of tumors examined by several of the current contributors in a previous study. ${ }^{16}$

\section{Definition of Tumor-Infiltrating Neutrophils}

Hematoxylin- and eosin-stained sections were examined under low power $(4 \times)$ to identify areas of neutrophilic aggregates within the tumor tissue. Only foci with neutrophils concentrated within and/or immediately adjacent to neoplastic cells were taken into consideration. Foci of inflammation with prominent stromal neutrophilia without preferential distribution of neutrophils around tumor epithelial cells, or areas immediately adjacent to ulcerations or necrosis, and those with foreign-body giant-cell reactions, suture granulomas or granulation tissue were disregarded.

\section{Tumor-infiltrating Neutrophil Count}

In cases with tumor-infiltrating neutrophils identified by $4 \times$ screening, 20 non-overlapping high power fields $(40 \times)$ were examined in representative areas on 2 slides of a given tumor (ie, a total of 40 fields per neoplasm). The number of tumor-related neutrophils was assessed in a semiquantitative manner using the mean value of high power fields in a $40 \times$ objective (magnification $400 \times ; 0.08 \mathrm{~mm}^{2}$ ). Areas with $\leq 10$ neutrophils/100 epithelial cells were considered negative and areas with $>10$ neutrophils/100 epithelial cells were considered as positive for tumor-infiltrating neutrophils, similar to that done in another study investigating this issue in gastric carcinoma. ${ }^{8}$ Positive areas were, then, further subdivided into two groups. Those with 11-15 tumor-infiltrating neutrophils/100 epithelial cells were designated as 'borderline' while those with $>15$ tumor-infiltrating neutrophils/100 epithelial cells were regarded as 'significant'.

The cutoffs used for tumor-infiltrating neutrophils were similar, albeit not identical, to those used in another study examining the same phenomenon in gastric carcinomas. ${ }^{8}$ Furthermore, we found that when tumor-infiltrating neutrophils were present they were quite evident. There were very few incidental neutrophils (focal, and in the range of 1-2/100 epithelial cells) and when they were present they were typically $>10 / 100$ epithelial cells.

\section{Statistical Analysis}

To examine the association of pancreatic tumor types with tumor-infiltrating neutrophil positivity, Fisher's Exact tests were performed owing to the small sample size in some groups. Additionally, the association of tumor-infiltrating neutrophils and specific solid and cystic pancreatic neoplasms (usual ductal carcinoma, micropapillary carcinoma, undifferentiated carcinoma, pancreatobiliary mucinous cystic neoplasms with papillary carcinoma in situ and intraductal papillary mucinous neoplasms) was compared using Fisher's Exact tests. All statistical significance was assessed using an $\alpha$ level of 0.05 . 


\section{MUC1 (Cell Surface-Associated Mucin 1) Labeling Profile}

Several previous reports have implicated mucin 1 (MUC1) in the immune response mediation of neoplastic cells. ${ }^{17,18}$ Therefore, once our study results showed that tumor-infiltrating neutrophils had preferential distribution toward certain tumor types, which are known also to have a tendency to express mucin 1, cell surface associated (MUC1), we decided to further investigate any possible association by expanding the study to include testing for MUC1 immunohistochemical expression by these tumor types.

Therefore, a select group of tumors from each category was immunolabeled with antibodies to mucin 1, cell surface associated (MUC1) (clone Ma695, Novocastra, Newcastle, UK) at a dilution of 1:100 in citrate and with an incubation period of $2 \mathrm{~h}$. Breast carcinoma tissue was used as a positive control.

\section{Results}

\section{In Invasive Carcinomas}

Among 363 invasive ductal carcinomas, 18 were positive for tumor-infiltrating neutrophils (15 significant and 3 borderline). Neutrophils were either intraepithelial or concentrated in the stroma immediately adjacent to the epithelium. Focal clustering of neutrophils (microabscess formation) was also present. The tumor-infiltrating neutrophils demonstrated a striking tumoro-centric distribution, being most concentrated within the denser areas of neoplastic cells and progressively decreasing in number away from the neoplasm.

Eight of the neutrophil-positive invasive ductal carcinomas were of micropapillary type (Figure 1) and the remaining seven were undifferentiated carcinomas (Figure 2). The cases with borderline tumor-infiltrating neutrophils had a focal micropapillary pattern.

Tumor-infiltrating neutrophils were not detected in ordinary tubule-forming ductal adenocarcinomas or other types of invasive cancer. Only 3 of 14 colloid carcinomas had borderline tumor-infiltrating neutrophils but these were predominantly in the mucin lakes rather than in the vicinity of the neoplastic epithelial cells (Figure 3) and thus disregarded. No borderline or significant tumorinfiltrating neutrophils were noted in nine acinar cell carcinomas.

\section{In Pre-Invasive Neoplasms}

Among pre-invasive neoplasms, tumor-infiltrating neutrophils appeared to have a predilection for those with pancreatobiliary cell lineage (Figures 4-7): significant tumor-infiltrating neutrophils were identified in 11/19 (58\%) mucinous cystic neoplasms with
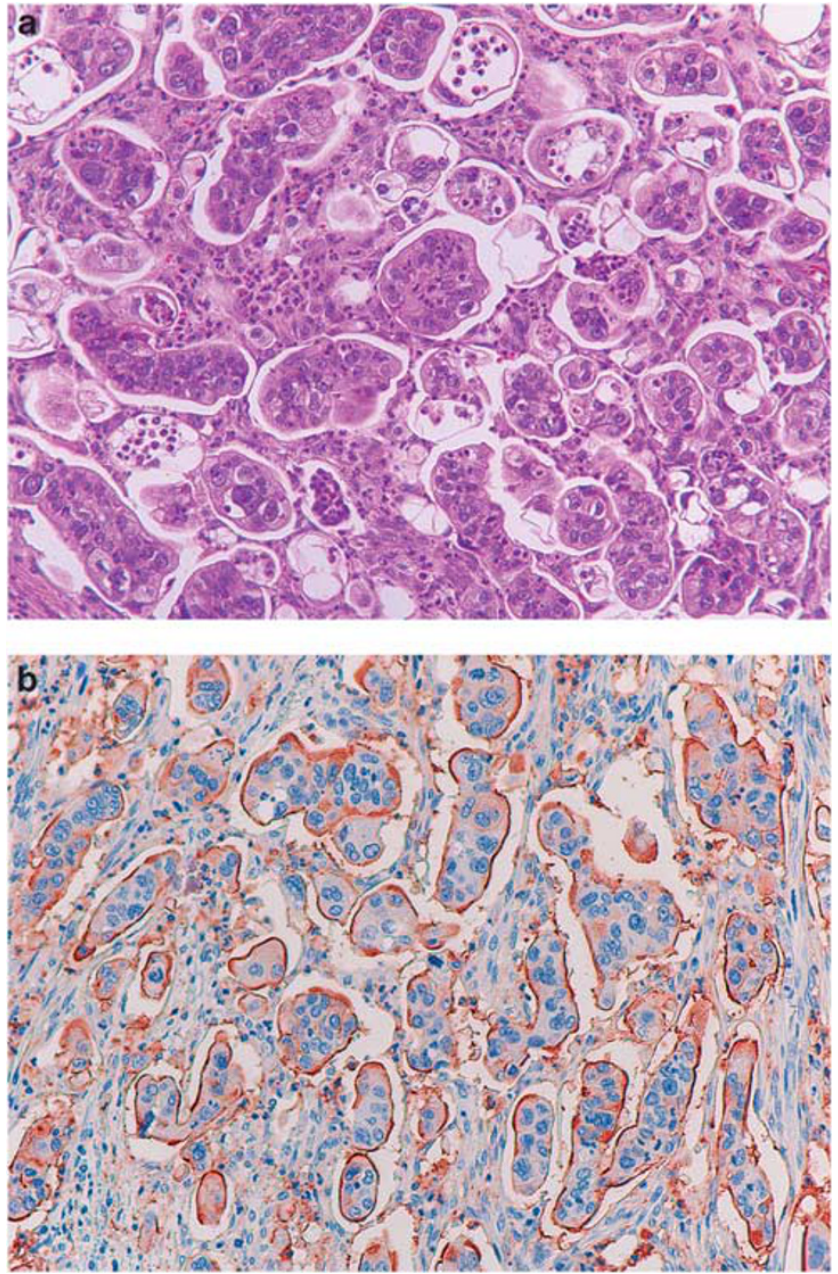

Figure 1 (a) Invasive micropapillary carcinoma composed of clusters of tumor cells within cleft-like spaces, characteristic inverse cell polarity and abundant intraepithelial and stromal tumor-infiltrating neutrophils (hematoxylin and eosin stain, magnification $\times 200$ ). (b) MUC1 staining of the stroma-facing surface of cell clusters in invasive micropapillary carcinoma (magnification $\times 200)$.

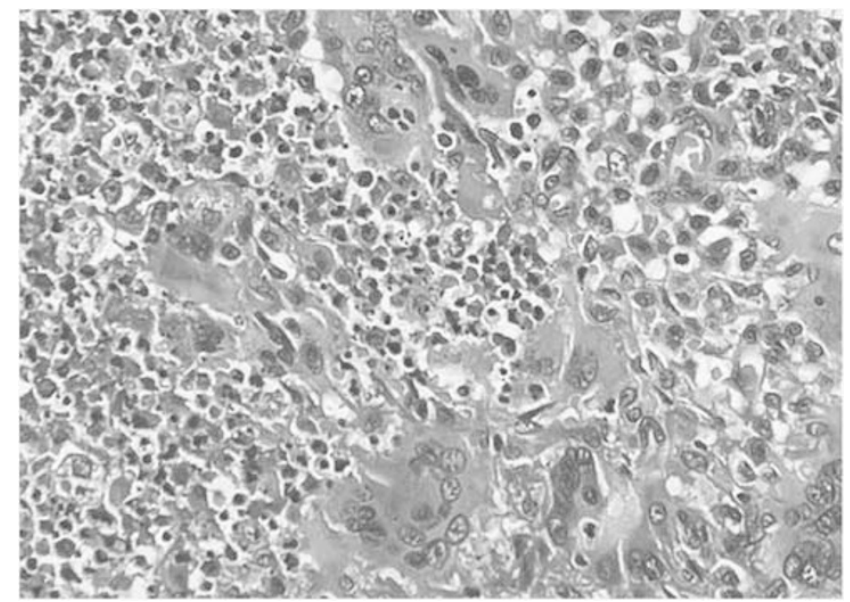

Figure 2 Undifferentiated carcinoma with prominent osteoclastlike giant cells, resembling osteoclastic giant cell carcinoma. Note significant numbers of tumor-infiltrating neutrophils within the stroma (hematoxylin and eosin stain, magnification $\times 200$ ). 


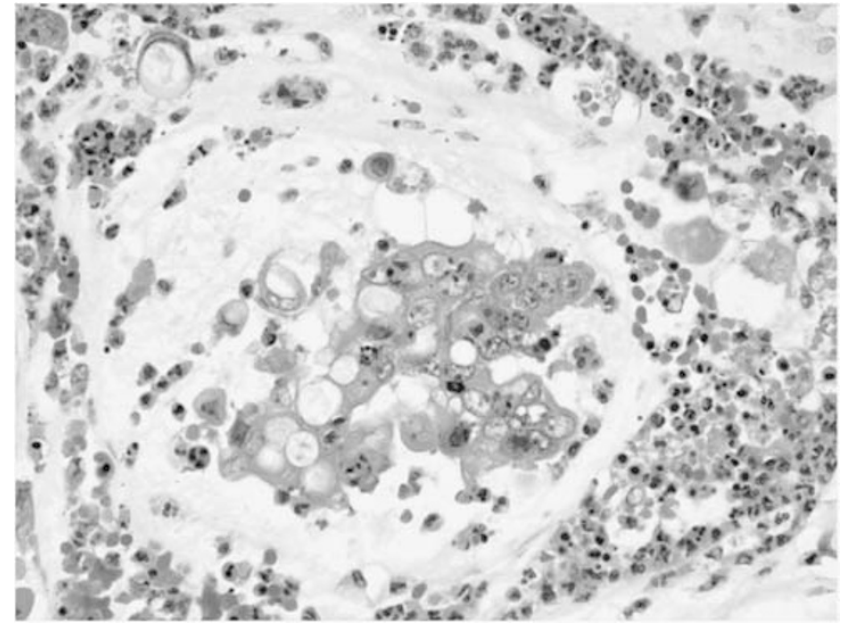

Figure 3 Colloid carcinoma of pancreas with abundant extracellular mucin and borderline numbers of intraepithelial and background tumor-infiltrating neutrophils (hematoxylin and eosin stain, magnification $\times 400$ ).

papillary carcinoma in-situ component (all of these had pancreatobiliary differentiation) (Figures 4 and 5) as well as in $4 / 16(25 \%)$ intraductal papillary mucinous neoplasms with pancreatobiliary-type papillae (Figures 6 and 7). However, among the intraductal papillary mucinous neoplasms with other types of papillae, only $1 / 11$ oncocytic type and $1 / 23$ intestinal type had borderline tumor-infiltrating neutrophils and all 10 gastric types were negative for tumorinfiltrating neutrophils. In cases that were positive for tumor-infiltrating neutrophils, the neutrophils were concentrated around the papillary areas, being typically present diffusely throughout the papillary component (Figures 4-6), without any significant involvement of either the non-papillary epithelium or the stroma away from the papillae. Low-grade and non-papillary (flattened) components of mucinous cystic neoplasms and intraductal papillary mucinous neoplasms failed to show tumor-infiltrating neutrophils.

\section{Other Tumor Types}

None of the 18 serous cystadenomas, 40 neuroendocrine tumors or 8 solid-pseudopapillary neoplasms were positive for tumor-infiltrating neutrophils.

\section{MUC1 Immunolabeling Profile}

MUC1 immunolabeling profile among tumors showed significant correlation with distribution of tumor-infiltrating neutrophils. Micropapillary carcinomas of the pancreas revealed diffuse and strong positivity for MUC1, which was predominantly on the stroma-facing (basal) surface of the micropapillary clusters, as a thin band of labeling (Figure 1b). Similarly, undifferentiated carcinomas showed uniformly strong staining with MUC1. More interestingly,
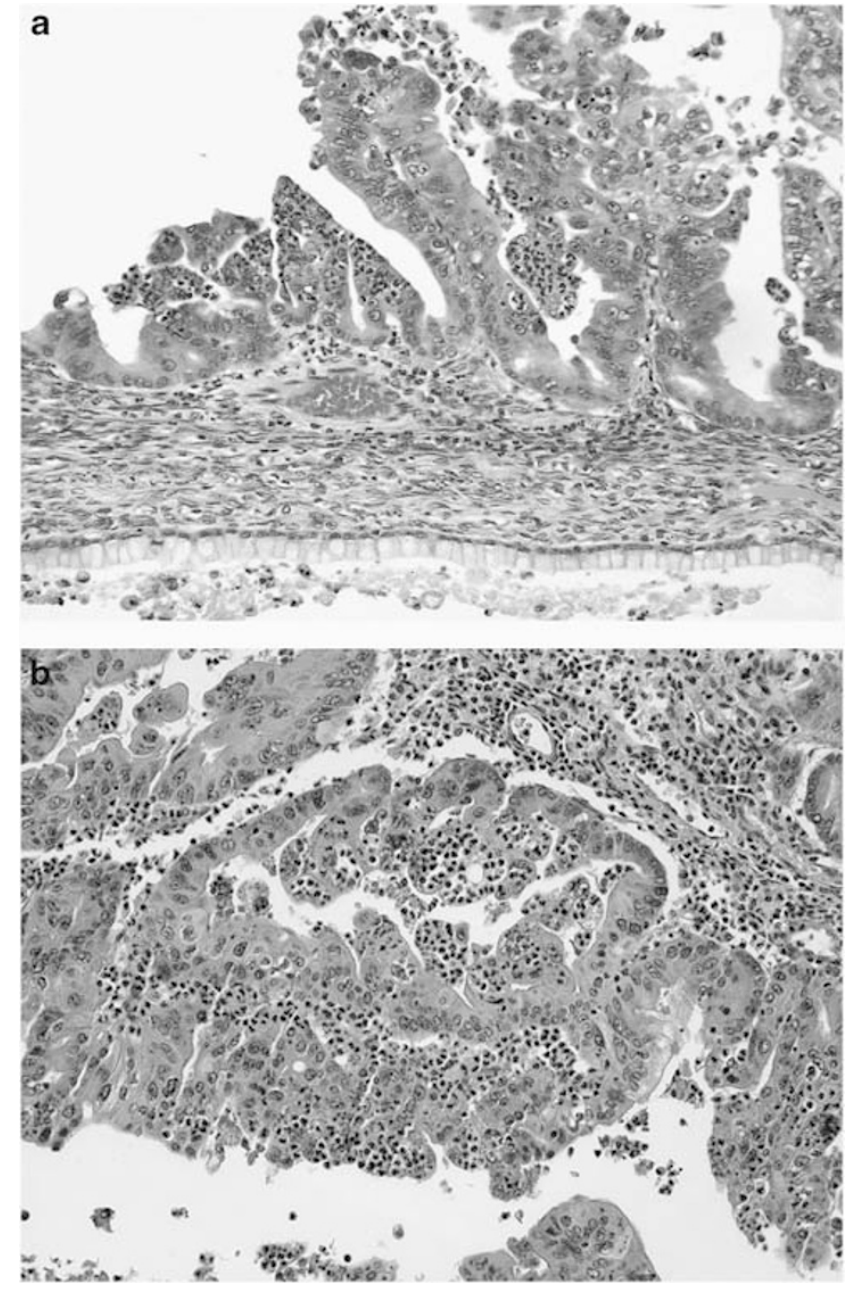

Figure 4 (a) Mucinous cystic neoplasm with bland mucinous columnar epithelium (at bottom), underlying ovarian-like stroma and papillary carcinoma in-situ (at top). Neutrophils are condensed around papillae and are both intra- and extraepithelial (hematoxylin and eosin stain, magnification $\times 200$ ). (b) Mucinpoor variant of mucinous cystic neoplasm with papillary carcinoma in-situ containing significant numbers of intra- and extraepithelial neutrophils (hematoxylin and eosin stain, magnification $\times 200$ ).

the mucinous cystic neoplasms with papillary in-situ carcinoma component and the intraductal papillary mucinous neoplasms with pancreatobiliary-type papillae all strongly expressed MUC1, which was confined to the surface of the papillae, as a thin band of labeling (Figures $5 \mathrm{~b}$ and $7 \mathrm{~b}$ ).

The results of analysis of all 517 pancreatic tumors are summarized in Table 1.

\section{Results of Statistical Analysis}

On comparison of usual ductal adenocarcinoma with micropapillary and undifferentiated carcinoma, there was a statistically significant difference between the number of tumor-infiltrating neutrophil-positive and -negative tumors, with micropapillary and 

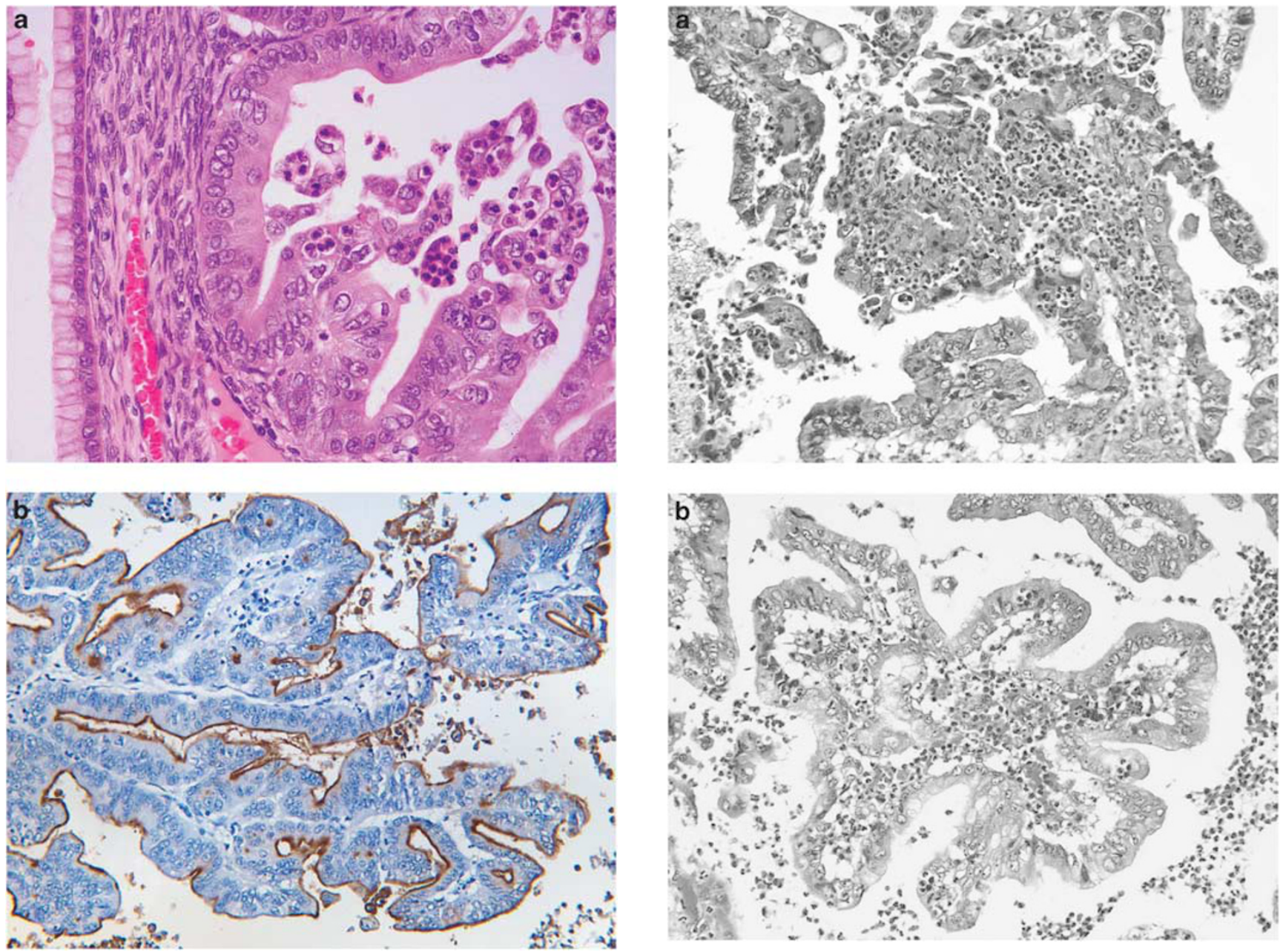

Figure 5 Papillary in-situ carcinoma in mucinous cystic neoplasm. (a) The neoplastic epithelium contains abundant intraepithelial neutrophils with focal microabscess formation (hematoxylin and eosin stain, magnification $\times 400$ ). (b) Note MUC1 expression on the apices of the papillae (magnification $\times 200$ ).

undifferentiated carcinomas showing significantly more positivity than usual ductal carcinoma $(P<0.0001)$. The pancreatobiliary mucinous cystic neoplasms with high-grade dysplasia and the pancreatobiliary-type intraductal papillary mucinous neoplasms were also significantly more likely to show tumor-infiltrating neutrophil positivity than all other types of intraductal papillary mucinous neoplasms $(P<0.0001)$. The results of statistical analysis of the tumor-infiltrating neutrophil-positive tumors are summarized in Table 2 .

\section{Discussion}

This study suggests that preferential chemotaxis of neutrophils to certain types of neoplasms is seen in the pancreas, and that this phenomenon occurs in a tumor-type- and cell-lineage-dependent fashion. Among invasive pancreatic carcinomas, tumor-

Figure 6 (a) Pancreatobiliary-type intraductal papillary mucinous neoplasm with complex papillae lined by mucin-containing cells and (b) abundant intraepithelial and stromal neutrophils concentrated within papillae (hematoxylin and eosin stain, magnification $\times 200$ ).

infiltrating neutrophils occur almost exclusively in micropapillary or undifferentiated carcinomas, and among pre-invasive neoplasia, tumor-infiltrating neutrophils occur preferentially in the neoplasms with pancreatobiliary-type lineage, detected in a significant proportion of the mucinous cystic neoplasms (in their in-situ papillary carcinoma component) and in a lesser degree of pancreatobiliary-type intraductal papillary mucinous neoplasms.

These findings beg the question 'why should tumor-infiltrating neutrophils show a predilection for invasive micropapillary pancreatic carcinoma, as well as mucinous cystic neoplasms and intraductal papillary mucinous neoplasms with in-situ papillary carcinomas and 'pancreatobiliary' lineage?'. It is safe to assume that these histologic subsets somehow act as chemoattractants for acute inflammatory cells. It is intriguing that the cellular morphology of the tumor-infiltrating neutrophil-positive carcinomas shows a common characteristic, in that all 

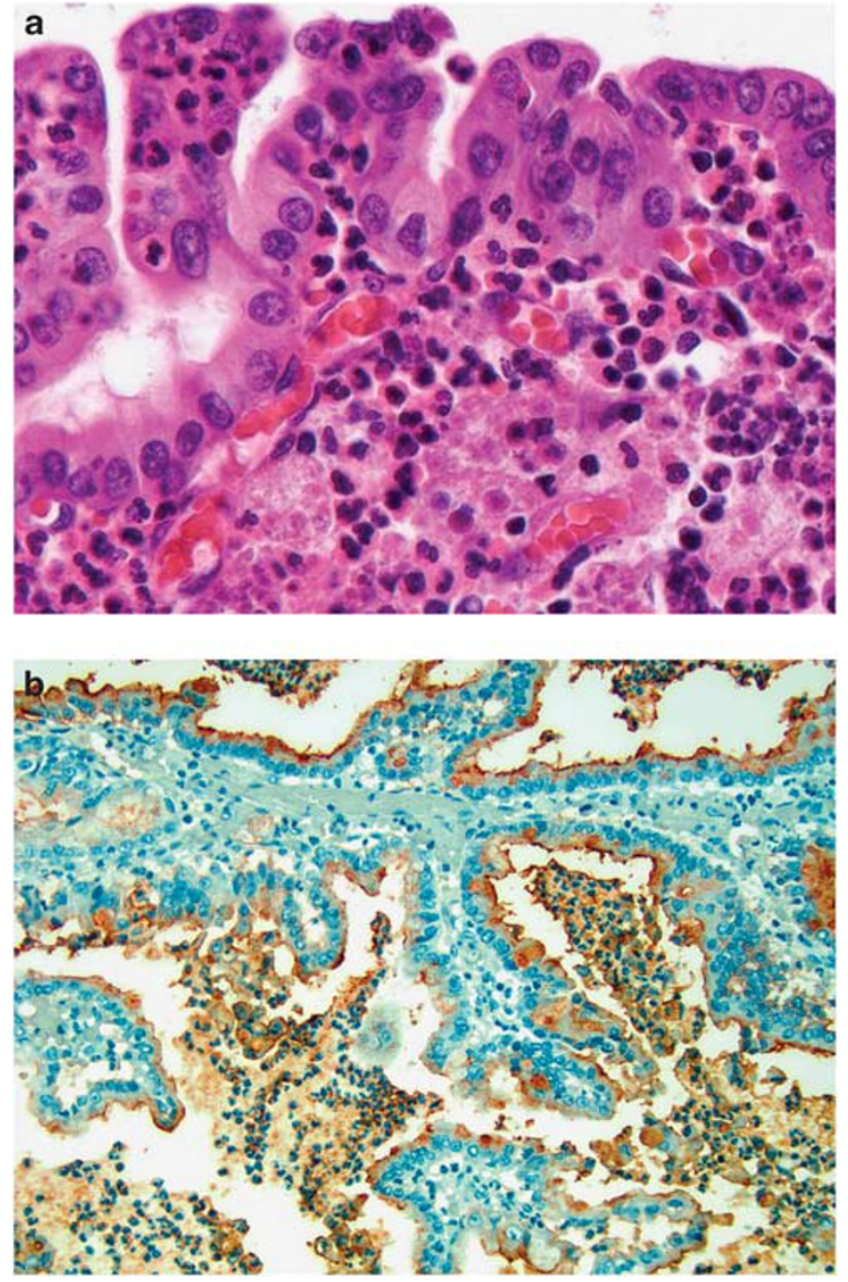

Figure 7 (a) High-power view of neutrophils concentrated within and adjacent to neoplastic cells in pancreatobiliary-type intraductal papillary mucinous neoplasm (hematoxylin and eosin stain, magnification $\times 400$ ). (b) Intraductal papillary mucinous neoplasm with pancreatobiliary-type papillae expressing MUC1 on the apical aspects of the neoplastic papillae (magnification $\times 200)$.

of them are mucin-depleted versions of their respective categories. Conventional tubule-forming adenocarcinomas, which tend to have significant amounts of intracellular mucin, do not typically show tumor-infiltrating neutrophils, whereas micropapillary and undifferentiated ones, which are relatively mucin-poor, do. Similarly, among preinvasive neoplasms, intestinal or gastric-type intraductal papillary mucinous neoplasms, which are characterized by copious mucin production, also appear to somehow distract tumor-infiltrating neutrophils; in contrast, pancreatobiliary variant does not. Along the same lines, metabolic activity of tumor-infiltrating neutrophil-attractant cells may also have some common features as evidenced by their morphology. The nuclei are typically cuboidalto-ovoid and have distinctive washed-out chromatin and small nucleoli, and additionally, the cytoplasm is relatively abundant despite the high-grade cytology. This is something we have also noted in
Table 1 List of pancreatic neoplasms analyzed for tumorinfiltrating neutrophils

\begin{tabular}{|c|c|c|c|}
\hline Pancreatic tumors $(\mathrm{n}=517)$ & $\begin{array}{c}\leq 10 \\
T^{\prime} N s^{\mathrm{a}}\end{array}$ & $\begin{array}{l}11-15 \\
T^{2} N s^{\mathrm{a}}\end{array}$ & $\begin{array}{l}>15 \\
\text { TINS }\end{array}$ \\
\hline \multicolumn{4}{|l|}{ Invasive ductal carcinomas $(\mathrm{n}=363)$} \\
\hline Usual ductal carcinoma $(n=340)$ & 340 & 0 & 0 \\
\hline Micropapillary carcinoma $(n=16)$ & 5 & 3 & 8 \\
\hline Undifferentiated carcinoma $(n=7)$ & 0 & 0 & 7 \\
\hline Acinar cell carcinoma $(n=9)$ & 9 & 0 & 0 \\
\hline Mucinous cystic neoplasms $(n=19)$ & 8 & 0 & 11 \\
\hline \multicolumn{4}{|c|}{ Intraductal papillary mucinous neoplasms $(\mathrm{n}=60)$} \\
\hline Pancreatobiliary type $(n=16)$ & 12 & 0 & 4 \\
\hline Intestinal type $(n=23)$ & 22 & 1 & 0 \\
\hline Oncocytic type $(n=11)$ & 10 & 1 & 0 \\
\hline Gastric type $(n=10)$ & 10 & 0 & 0 \\
\hline Serous cystadenomas $(n=18)$ & 18 & 0 & 0 \\
\hline Neuroendocrine neoplasms $(n=40)$ & 40 & 0 & 0 \\
\hline Solid-pseudopapillary neoplasms $(n=8)$ & 8 & 0 & 0 \\
\hline
\end{tabular}

${ }^{\mathrm{a}}$ Tumor-infiltrating neutrophils/100 epithelial cells.

Table 2 Comparison of TIN-positive and TIN-negative pancreatic tumors using Fisher's exact test

\begin{tabular}{lcrll}
\hline Pancreatic tumors & $\begin{array}{c}\text { TIN } \\
\text { positive }^{\mathrm{a}}\end{array}$ & $\begin{array}{c}\text { TIN } \\
\text { negative }^{\mathrm{a}}\end{array}$ & P-value \\
\hline Usual ductal carcinoma $(n=340)$ & 0 & 340 & $<0.0001$ \\
Micropapillary carcinoma $(n=16)$ & 11 & 5 & \\
Usual ductal carcinoma $(n=340)$ & 0 & 340 & $<0.0001$ \\
Undifferentiated carcinoma $(n=7)$ & 7 & 0 & \\
$\begin{array}{l}\text { MCN }(n=19) \text { and IPMN- } \\
\text { pancreatobiliary type }(n=16)\end{array}$ & 15 & 20 & $<0.0001$ \\
IPMN-all other subtypes $(n=44)$ & 2 & 42 & \\
\hline
\end{tabular}

${ }^{\mathrm{a}}$ Tumor-infiltrating neutrophils/100 epithelial cells.

MCN, mucinous cystic neoplasms; IPMN, intraductal pancreatic mucinous neoplasms.

colorectal adenocarcinomas showing tumor-infiltrating neutrophils (unpublished observations).

Interestingly, most of the pancreatic neoplasms that preferentially attract tumor-infiltrating neutrophils also differentially express MUC1 (both in the stroma-facing surfaces of cells in the micropapillary carcinomas and on the surfaces of dysplastic papillae in pancreatobiliary-type mucinous cystic neoplasm and intraductal papillary mucinous neoplasm). This phenomenon of MUC1 expression as confirmed in this study, has also been previously observed in invasive micropapillary pancreatic carcinoma, ${ }^{16,19}$ pancreatic ductal carcinoma, ${ }^{20,21}$ as well as the pancreatobiliary variant of intraductal papillary mucinous neoplasm. ${ }^{22}$

MUC1 is a large transmembrane glycoprotein that is expressed on the apex of ductal epithelial cells, and has been said to have a role in spatial orientation of cells, including the maintenance of lumen formation. ${ }^{23}$ MUC1 is known to ${ }^{24}$ promote inflammation ${ }^{25}$ by acting as a powerful chemoattractant for the innate immune system ${ }^{17,26-27}$ and 
reported to drive the progression to colitis-associated colon cancer ${ }^{17}$ through cytokine and oxygen metabolite production. ${ }^{21,22}$

Considering the established role of MUC1 in immune modulation by tumor cells and also the parallels in the expression profile of this molecule in different subsets of pancreatic neoplasia, it is tempting to think that it may have a role in the recruitment of neutrophils to some tumor types, namely micropapillary and undifferentiated carcinomas, as well as in the in-situ papillary carcinoma components of mucinous cystic neoplasms and pancreatobiliary variant of intraductal papillary mucinous neoplasms; however, how would we then explain the absence of tumor-infiltrating neutrophils in the other 340 conventional ductal adenocarcinomas we tested, which are also known to express MUC1. ${ }^{20-21,24,28}$ For this, one can speculate that the degree of exposure of MUC1 to the external milieu, in particular to the stroma, may be an important factor. In ordinary invasive ductal adenocarcinomas, MUC1 is typically expressed in the apical membrane of the infiltrating units, and as such it is not as readily exposed to the stroma or external milieu. However, in micropapillary carcinomas there is a characteristic inverse cell polarity in which MUC1 is expressed in the stroma-facing surface of the cells. ${ }^{19}$ Similarly, most undifferentiated carcinomas have MUC1 expressed in their membranes that readily interact with the stroma. Therefore, it is conceivable that MUC1-stroma interaction may be a factor in the recruitment of the neutrophils, which has been suggested in some studies ${ }^{25-27}$ however, undoubtedly, there are several other contributing tumor-specific factors that have a role in this as well.

Tumor-infiltrating neutrophils may reflect a natural cytotoxity of the host against the tumors or may be the result of cytokine production by the tumor cells. Whatever the cause, one resulting effect of any tumor-related immunological host response would be overall patient survival. Some studies have shown that tumor-infiltrating neutrophils cause tumor destruction by releasing cytotoxic mediators and promoting cell-mediated cytotoxicity. ${ }^{1-3}$ Also, increased blood neutrophils have been shown to reduce the risk of metastasis in lymphoma, melanoma and lung carcinoma cell lines. ${ }^{15}$ Tumorinfiltrating neutrophils have been associated with reduced mortality risk in females with gastric carcinoma in one study. ${ }^{8}$ Yet, other studies have shown that tumor-infiltrating neutrophils have no significant effect on prognosis in either gastric ${ }^{10}$ or colorectal cancer. ${ }^{13}$ Conversely, tumor-infiltrating neutrophils have been shown to increase the metastatic potential of mammary ${ }^{4-5}$ and bronchioloalveolar carcinoma. ${ }^{6,12}$

Similarly, the poorer prognosis and shortened survival associated with micropapillary carcinoma may in part be related to the presence of abundant tumor-infiltrating neutrophils and their promotion of tumor cell growth and metastasis. Additionally, this tumor's coexpression of MUC1, which has carcinogenic properties, has been previously established in the pancreas ${ }^{18,21-22,24}$ and other organs. MUC1 promotes cell proliferation, metastasis and immunoresistance, and inhibits cell-cell and cellstroma interaction in pancreatic neoplasms making it easier for tumor cells to dissect the stroma. ${ }^{18}$ Its' inhibitory role in cell-stroma interaction may be a key factor in the detachment of tumor cells from the stroma, thus resulting in the papillary morphology seen in these neutrophil-rich tumors. MUC1 is also expressed in high-grade pancreatic intraepithelial neoplasia and is believed to contribute to the dysplasia-carcinoma sequence in pancreatic tumors. ${ }^{21}$ The concomitant expression of tumorinfiltrating neutrophils and MUC1 by this subset of pancreatic neoplasms, with the two participating in a vicious cycle, may promote more aggressive behavior in these tumors (all of which, interestingly, showed either in-situ or invasive carcinoma). However, as interesting as this potential association may appear, such a relationship has not been established and deserves to be investigated in a larger population cohort.

The predilection of tumor-infiltrating neutrophils for a certain subset of mucinous neoplasms including pancreatic mucinous cystic neoplasms, intraductal papillary mucinous neoplasms and even gynecologic tumors such as endocervical-like mucinous borderline tumors of the ovary (in which tumor-infiltrating neutrophils are part of the diagnostic criteria) $^{29}$ also deserves mention. Is the mucin produced by these tumors proinflammatory and if so why does the colloid subtype of pancreatic adenocarcinoma not express tumor-infiltrating neutrophils as well? Clearly, other factors independent of mucin production are at play in this group of mucinous neoplasms.

In summary, tumor-infiltrating neutrophils are seen in a small subset of pancreatic neoplasms including micropapillary carcinoma, undifferentiated carcinoma, mucinous cystic neoplasm with in-situ papillary carcinoma and pancreatobiliarytype intraductal papillary mucinous neoplasm with in-situ carcinoma. Whether the neutrophils are secondarily recruited by these specific histologic groups of tumors or are primarily participating in their development is uncertain. It is also unclear whether the presence of tumor-infiltrating neutrophils in these tumors has beneficial or detrimental effects on tumor behavior. It is noteworthy that all of these neutrophil-rich pancreatic tumors also expressed MUC1, a powerful chemoattractant and marker of aggressive behavior.

\section{Acknowledgement}

We are indebted to Dr Charles Parkos of the Department of Pathology, Emory University, for his critical review of the manuscript and invaluable suggestions. This study was supported in part by the 
National Cancer Institute Specialized Program in Research Excellence (SPORE) CA101936 in Pancreas Cancer (PAR-02-068) and in part by the Georgia Cancer Coalition Distinguished Cancer Clinicians and Scientists Program.

\section{Disclosure/conflict of interest}

The authors declare no conflict of interest.

\section{References}

1 Di Carlo E, Forni G, Lollini P, et al. The intriguing role of polymorphonuclear neutrophils in antitumor reactions. Blood 2001;97:339-345.

2 Dallegri F, Ottonello L, Ballestrero A, et al. Tumor cell lysis by activated human neutrophils: analysis of neutrophil-delivered oxidative attack and role of leukocyte function-associated antigen 1. Inflammation 1991;15:15-30.

3 Kindzelskii AL, Petty HR. Early membrane rupture events during neutrophil-mediated antibody-dependent tumor cell cytolysis. J Immunol 1999;162:3188-3192.

4 Aeed PA, Nakajima M, Welch DR. The role of polymorphonuclear leukocytes (PMN) on the growth and metastatic potential of $13762 \mathrm{NF}$ mammary adenocarcinoma cells. Int J Cancer 1988;42:748-759.

5 Welch DR, Schissel DJ, Howrey RP, et al. Tumorelicited polymorphonuclear cells, in contrast to 'normal' circulating polymorphonuclear cells, stimulate invasive and metastatic potentials of rat mammary adenocarcinoma cells. Proc Natl Acad Sci U S A 1989; 86:5859-5863.

6 Bellocq A, Antoine M, Flahault A, et al. Neutrophil alveolitis in bronchioloalveolar carcinoma: induction by tumor-derived interleukin-8 and relation to clinical outcome. Am J Pathol 1998;152:83-92.

7 Apple SK. Invasive ductal carcinoma with tissue neutrophilia: a distinct entity? Breast (Edinburgh, Scotland) 2004;13:519-521.

8 Caruso RA, Bellocco R, Pagano M, et al. Prognostic value of intratumoral neutrophils in advanced gastric carcinoma in a high-risk area in northern Italy. Mod Pathol 2002;15:831-837.

9 Fossati G, Ricevuti G, Edwards SW, et al. Neutrophil infiltration into human gliomas. Acta Neuropathologica 1999;98:349-354.

10 Rice AJ, Griffiths AP, Martin IG, et al. Gastric carcinoma with prominent neutrophil infiltration. Histopathology 2000;37:289-290.

11 Wislez M, Fleury-Feith J, Rabbe N, et al. Tumorderived granulocyte-macrophage colony-stimulating factor and granulocyte colony-stimulating factor prolong the survival of neutrophils infiltrating bronchoalveolar subtype pulmonary adenocarcinoma. Am J Pathol 2001;159:1423-1433.

12 Wislez M, Rabbe N, Marchal J, et al. Hepatocyte growth factor production by neutrophils infiltrating bronchioloalveolar subtype pulmonary adenocarcinoma: role in tumor progression and death. Cancer Res 2003;63:1405-1412.

13 Nielsen HJ, Hansen U, Christensen IJ, et al. Independent prognostic value of eosinophil and mast cell infiltration in colorectal cancer tissue. I Pathol 1999;189:487-495.

14 Musiani P, Allione A, Modica A, et al. Role of neutrophils and lymphocytes in inhibition of a mouse mammary adenocarcinoma engineered to release IL-2, IL-4, IL-7, IL-10, IFN-alpha, IFN-gamma, and TNF-alpha. Lab Invest 1996;74:146-157.

15 Matsumoto Y, Saiki I, Murata J, et al. Recombinant human granulocyte colony-stimulating factor inhibits the metastasis of hematogenous and non-hematogenous tumors in mice. Int J Cancer 1991;49:444-449.

16 Khayyata S, Basturk O, Adsay NV. Invasive micropapillary carcinomas of the ampullo-pancreatobiliary region and their association with tumor-infiltrating neutrophils. Mod Pathol 2005;18:1504-1511.

17 Beatty PL, Plevy SE, Sepulveda AR, et al. Cutting edge: transgenic expression of human MUC1 in IL-10-/- mice accelerates inflammatory bowel disease and progression to colon cancer. J Immunol 2007;179:735-739.

18 Satoh S, Hinoda Y, Hayashi T, et al. Enhancement of metastatic properties of pancreatic cancer cells by MUC1 gene encoding an anti-adhesion molecule. Int J Cancer 2000;88:507-518.

19 Nassar H, Pansare V, Zhang $\mathrm{H}$, et al. Pathogenesis of invasive micropapillary carcinoma: role of MUC1 glycoprotein. Mod Pathol 2004;17:1045-1050.

20 Westgaard A, Schjolberg AR, Cvancarova M, et al. Differentiation markers in pancreatic head adenocarcinomas: MUC1 and MUC4 expression indicates poor prognosis in pancreatobiliary differentiated tumours. Histopathology 2009;54:337-347.

21 Adsay NV, Merati K, Andea A, et al. The dichotomy in the preinvasive neoplasia to invasive carcinoma sequence in the pancreas: differential expression of MUC1 and MUC2 supports the existence of two separate pathways of carcinogenesis. Mod Pathol 2002;15:1087-1095.

22 Adsay NV, Merati K, Basturk O, et al. Pathologically and biologically distinct types of epithelium in intraductal papillary mucinous neoplasms: delineation of an 'intestinal' pathway of carcinogenesis in the pancreas. Am J Surg Pathol 2004;28:839-848.

23 Datta A, Bryant DM, Mostov KE. Molecular regulation of lumen morphogenesis. Curr Biol 2011;21:R126-R136.

24 Levi E, Klimstra DS, Andea A, et al. MUC1 and MUC2 in pancreatic neoplasia. J Clin Pathol 2004;57:456-462.

25 Kadayakkara DK, Beatty PL, Turner MS, et al. Inflammation driven by overexpression of the hypoglycosylated abnormal mucin 1 (MUC1) links inflammatory bowel disease and pancreatitis. Pancreas 2010;39:510-515.

26 Ryan SO, Vlad AM, Islam K, et al. Tumor-associated MUC1 glycopeptide epitopes are not subject to selftolerance and improve responses to MUC1 peptide epitopes in MUC1 transgenic mice. Biol Chem 2009;390:611-618.

27 Carlos CA, Dong HF, Howard OM, et al. Human tumor antigen MUC1 is chemotactic for immature dendritic cells and elicits maturation but does not promote Th1 type immunity. J Immunol 2005;175:1628-1635.

28 Yonezawa S, Higashi M, Yamada N, et al. Significance of mucin expression in pancreatobiliary neoplasms. J Hepato-Biliary-Pancreat Sci 2010;17:108-124.

29 Rodriguez IM, Irving JA, Prat J. Endocervical-like mucinous borderline tumors of the ovary: a clinicopathologic analysis of 31 cases. Am J Surg Pathol 2004;28:1311-1318. 